\title{
Linx
}

Revue des linguistes de l'université Paris X Nanterre

$44 \mid 2001$

Spécificité et histoire des discours sémiotiques

\section{Hjelmslev et Greimas : deux sémiotiques universelles différentes}

Driss Ablali

\section{(2) OpenEdition}

Journals

Édition électronique

URL : http://journals.openedition.org/linx/1031

DOI : 10.4000/linx.1031

ISSN : 2118-9692

Éditeur

Presses universitaires de Paris Nanterre

Édition imprimée

Date de publication : 1 juin 2001

Pagination : $39-53$

ISSN : 0246-8743

\section{Référence électronique}

Driss Ablali, «Hjelmslev et Greimas : deux sémiotiques universelles différentes », Linx [En ligne],

44 | 2001, mis en ligne le 05 juillet 2012, consulté le 19 avril 2019. URL : http://

journals.openedition.org/linx/1031; DOI : 10.4000/linx.1031

Ce document a été généré automatiquement le 19 avril 2019.

Département de Sciences du langage, Université Paris Ouest 


\title{
Hjelmslev et Greimas : deux sémiotiques universelles différentes
}

\author{
Driss Ablali
}

\begin{abstract}
à Anouar Ben Msila
Nous tenons à exprimer notre gratitude à M. Arrivé, notre directeur de thèse, à Cl. Normand, qui suit généreusement nos travaux, et à Fr. Gobert qui ont bien voulu lire notre manuscrit, nous faire bénéficier de leurs remarques et ainsi nous éviter bien des faux pas. Mais il va de soi que ce travail, en définitive, n'engage que nous seul.
\end{abstract}

1 Le terme de "sémiotique » exige souvent de laborieuses mises au point. Il se trouve actuellement au centre d'un remue-ménage qui agite les sciences du langage et qui s'étend même au dehors de celles-ci. Son intégration a lieu dans des champs de recherche si dissemblables qu'on ne saurait leur trouver un air de famille : ils ne se reconnaissent pas même entre eux. Cette dissemblance, certes, ne date pas d'hier. Rien ne permet, par exemple, de rapprocher la sémiotique de Peirce, qui prétend être une théorie de la connaissance - une logique, d'après l'intention de Peirce lui-même - de celle de Saussure ou de Greimas, qui se présentent comme des sémiotiques linguistiques ${ }^{1}$. N'est-ce pas alors prendre le parti de la complication, voire de la contradiction, que d'associer à la sémiotique des personnalités aussi contrastées que Locke, Peirce, Saussure, Hjelmslev, Benveniste, Greimas, Prieto, Lotman, pour ne citer qu'eux, ainsi qu'on a encore coutume de le faire?

2 Au départ, une opposition semble s'être faite entre la sémiotique européenne et la sémiotique américaine. En France, durant l'ère structuraliste, c'était surtout à Greimas qu'on pensait chaque fois que le terme de sémiotique était évoqué ; il fallait cependant faire une exception avec Perpignan, où G. Deledalle et son équipe travaillaient sur la sémiotique de Peirce. En revanche, aux États-Unis, où le terme est employé dans sa forme plurielle - semiotics ${ }^{2}$ - , ce sont les travaux de Peirce ou Morris qui permettent de s'en réclamer. Mais il est vrai que la sémiotique américaine reste peu connue en France, surtout dans le milieu des linguistes, et que sans doute l'introduction de cette sémiotique 
dans les programmes universitaires n'est pas pour demain. Aussi n'est-ce pas cette opposition inter-atlantique qui est la cause fondamentale de l'ambivalence du terme de sémiotique.

3 On constate en revanche que le terme de sémiotique revient ces derniers temps sous les plumes de chercheurs tels que Fr. J. Varela, P. Ricoeur, R. Thom, Fr. Rastier, B. Cyrulnik, J$\mathrm{Cl}$. Coquet, J. Fontanille ${ }^{3}$, qui sont loin de partager les mêmes objets de connaissance. Cette liste hétérogène suscite bien des réflexions. Bornons-nous à constater qu'aujourd'hui le terme de sémiotique ne caractérise plus personne. Autrement dit, il ne caractérise plus un domaine de recherche particulier: bien des chercheurs, travaillant dans des domaines de recherche divers, se l'approprient sans spécifier son sens.

Nous nous proposons d'étudier ici la coexistence au sein de la sémiotique européenne de deux paradigmes, attachés respectivement aux noms de Hjelmslev et de Greimas. Plus précisément, nous cherchons à savoir ce qu'il en est de la reconduction du projet glossématique de Hjelmslev dans la sémiotique de Greimas.

En effet, on place souvent Greimas dans la continuité de Hjelmslev, en invoquant des concepts comme texte, connotation, dénotation, immanence, empirisme, pour s'en tenir aux plus connus. Cette continuité mérite d'être examinée de plus près : les deux sémiotiques ne visent pas à recouvrir les mêmes types de problématiques, même si elles empruntent les mêmes voies. C'est ce que nous argumentons dans la première partie, introduite par quelques commentaires sur la réception tardive de l'œuvre de Hjelmslev, en interrogeant la notion de «texte » à travers la catégorisation «universel»vs «général». Dans la seconde partie, nous montrons que ce sentiment de parenté n'est pas pour autant sans fondement : entre Hjelmslev et Greimas, il y a bien une continuité. Seulement, il ne faut pas la chercher directement dans la reconduction par Greimas du projet linguistique de la glossématique, mais bien dans la réflexion épistémologique de Hjelmslev, lorsque celui-ci conçoit la sémiotique comme une sémiotique universelle avec des sémiotiques spécifiques.

\section{Hjelmslev : une réception tardive}

Arrivé commente ce paradoxe dans les termes suivants ${ }^{4}$ :

À qui jette un coup d'œil sur la production linguistico-méthodologique de la sémiotique contemporaine - voire d'une façon générale sur les discours des sciences humaines - apparaît à l'égard de la glossématique un trait caractéristique : à quelques rares exceptions, la référence à la glossématique est absente ou fortement péjorative (Arrivé 1981 : 305).

7 Hjelmslev n'a donc pas fait école. Son héritage, tant au Danemark qu'à l'étranger, n'a pas marqué beaucoup de linguistes. Dans son pays natal, contrairement à ce qui a eu lieu pour l'autre grand linguiste danois - V.Brøndal - le centenaire de sa naissance n'a pas été célébré5. La glossématique de Hjelmslev devait attendre le milieu des années quatre-vingt, grâce à l'intérêt des linguistes, notamment des sémioticiens, pour sortir d'une ombre de longue durée. Car si c'est Greimas qu'on désigne comme le véritable continuateur de Hjelmslev, ce sont des chercheurs de la même demeure, qu'on appelle l'École de Paris, ou parfois des ex-greimassiens, qui ont assuré le relais. On peut mettre au premier rang les travaux de Fr. Rastier, Cl. Zilberberg, M. Arrivé et H. Parret. En Italie, c'est sous le patronage d'un autre sémioticien, U. Eco, que, par des recherches, d'ordre épistémologique et exégétique, notamment grâce aux numéros de Protagora et Versus, va 
réapparaitre l'intérêt pour les écrits de Hjelmslev. Toujours en Italie, cet intérêt va jusqu'à faire naître le premier « Circolo glossematico » et une revue, Janus.

8 Mais, dans les années soixante-dix, la redécouverte en France de l'œuvre de Hjelmslev avait déjà été amorcée grâce à Greimas et à Barthes" ${ }^{6}$ Des textes comme "Éléments de sémiologie », Le système de la mode ou Sémantique structurale ont relancé l'intérêt pour les textes de Hjelmslev. Les deux sémioticiens ou sémiologues travaillaient, à cette époque, de conserve. À propos de cette période, citons la réponse de Greimas à une question posée par Arrivé lors du colloque qui lui était consacré à Cerisy-la-Salle :

Je n'arrive pas à me souvenir du moment de ma rencontre avec Hjelmslev. Je ne sais pas si c'est Barthes qui m'a dit que c'était important, ou si c'est moi qui l'ai dit à Barthes (1987 : 303).

9 Chacun aura sa façon d'aborder les textes de Hjelmslev. Les deux parcours de Greimas et de Barthes illustrent clairement le poids déterminant du Danois sur la naissance en France de deux sémiotiques différentes. Ici on peut lire avec intérêt que ces deux sémioticiens, s'ils sont partis tous deux des textes du Danois, n'ont pas privilégié le même point de départ :

Je sais que c'était un coup de foudre pour tous les deux [i.e. Greimas et Barthes].

Seulement, il a commencé par les Essais et moi par les Prolégomènes (1987 : 42).

Greimas a commencé par le chemin le plus théorique, préférant ainsi ce qu'il appelle le "système dénotatif », Barthes, lui, est allé vers les sémiotiques non scientifiques, c'est-àdire les «langages de connotation» (lesquels, cependant, contrairement à ce que laisse entendre Greimas, sont développés non dans les Essais, mais bien dans les Prolégomènes).

\section{Du texte à la langue vs du texte à la narrativité}

11 Par-delà ces divergences, l'apport novateur de Hjelmslev, son erreur géniale, pour reprendre une expression de Rastier, qui va beaucoup marquer la sémiotique en France, consiste à sortir de l'hypostase du signe : commencer non pas par les signes, mais par les relations, plus précisément par le texte :

La théorie du langage s'intéresse à des textes, et son but est d'établir une procédure permettant la description non contradictoire et exhaustive d'un texte donné (Hjelmslev $1943:$ 31).

Et encore ceci :

L'objet est bien entendu un texte que celui-ci se manifeste graphiquement sous la forme d'un message écrit ou phoniquement sous la forme d'un message oral (Hjelmslev 1985 : 77).

Greimas a bien retenu la leçon. Le signe ne mène nulle part, ce n'est qu'un point de départ, car le vrai travail commence sous les signes :

Pour moi, la science des signes, c'est une fausse définition. Parce que derrière les signes se cache le jeu des significations, et une analyse plus profonde amène à détruire, à déstructurer le signe, pour mettre à jour les univers de signification. Autrement dit, le travail de la sémiologie c'est de parvenir aux structures logicoabstraites sous-jacentes à l'effervescence des normes figuratives qui nous entourent,

explique Greimas dans un entretien accordé au journal Le Monde (1974).

13 Hjelmslev et Greimas, les sémioticiens connaissent cette bipolarité, mais il semble que la divergence entre ces deux pôles ait été sous-estimée. Car rares sont ceux parmi les linguistes qui sont allés lire le texte du Danois. Ce que l'on en connaît vient souvent de 
seconde main. Pour notre part, c'est en lisant un article de Cl. Zilberberg ${ }^{7}$, l'un des grands connaisseurs des textes hjelmsleviens, que nous avons décidé d'entrer dans les rouages de cette soi-disant continuité.

Le premier constat concerne le parcours des deux sémioticiens : Hjelmslev a voulu fonder une épistémologie pour toutes les sciences. Cela vaut la peine de rappeler immédiatement un passage central des Prolégomènes qui est à la base de notre point de vue :

Nous pensons qu'il est possible de supposer que plusieurs des principes généraux que nous avons été amenés à adopter au stade initial de la théorie du langage ne sont pas seulement valables pour la linguistique, mais pour toutes les sciences (1943: 17).

15 Avec ce geste, Hjelmslev, nous dit Rastier, voulait reformuler «à sa façon la thèse néopositiviste de l'unité de la science, que Morris et Carnap reprenaient en 1938 dans l' Encyclopaedia of Unified Science; mais il place cette unification sous l'égide de la linguistique et non de la logique » (Rastier $1997: 144)$.

Cette épistémologie générale justifie l'intérêt qu'a suscité l'œuvre de Hjelmslev dans des disciplines aussi différentes que la philosophie, avec Derrida, Deleuze, Ricoeur, Guattari, Eco, les sciences du langage, avec Martinet, Greimas, Rastier, Zilberberg, Parret, ou avec des théoriciens de la littérature, tels Barthes et Kristeva. Cette diversité explique les différentes interprétations de la sémiotique de Hjelmslev, comme nous le montre M. Rasmussen dans cet extrait :

Aussi voit-on en Hjelmslev un penseur à la fois positiviste (Gregerson 1991 : I, 28; II.214; Olsen 1989: 49), en tous cas pas positiviste (Mortensen 1972: 49; Kemp 1972: 133-34), idéaliste (Mounin 1965: 82), conceptualiste (Leroy 1952: 529), positiviste logique (Pavel $1988: 88$ ), néorationaliste (Barilli $1974: 126)$, platonicien (Jensen 1981: 88), défenseur d'un empirisme sceptique (Stjernfelt 1992: 184) ou bien d'une science à la fois aristotélicienne et galiléenne (Ranulf $1946: 186-96)$ voire comme «le métaphysicien d'un structuralisme» (Meschonnic 1975: 231). (Rasmussen 1993 : 115).

Ces différentes interprétations trouvent leurs explications par l'ambition même du projet glossématique de Hjelmslev. Il suffit de lire les premières pages des Prolégomènes pour s'en apercevoir, ou la dernière phrase :

La théorie du langage a ainsi atteint le but qu'elle s'était assigné : humanitas et universitas (1943: 171).

Greimas, lui, voulait construire, à travers le principe de la narrativité, une sémiotique qui ne tient pas compte du genre des textes : c'est-à-dire que rien ne devait échapper aux modèles construits par la sémiotique. Nous pensons à des notions comme "schéma actantiel ", "schéma narratif », ou le fameux "carré sémiotique ». L'heuristique de la sémiotique du discontinu se voulait universelle. Car le sens réside partout où il y a activité humaine, il est structuré d'une façon narrativement indépendante; ainsi la sémiotique retrouve les mêmes structures profondes, stables et universelles, sans tenir compte de la spécificité des discours. Les auteurs du Dictionnaire de sémiotique vont jusqu'à considérer les structures sémio-narratives comme des « formes de l'intelligence humaine $»^{8}$. Nous les avons vues appliquées au discours littéraire : le Maupassant de Greimas, les analyses d'Arrivé sur l'œuvre de Jarry, ou encore les recherches de Fontanille sur Proust en témoignent ; au discours juridique à travers les travaux de É. Landowski ; au discours scientifique avec les analyses de F. Bastide. Pour cette sémiotique, tout est narrativisable, aussi bien les textes verbaux que les textes non verbaux. Les analyses de F. Thürlemann ou de J-M. Floch sur la peinture ou la publicité, ou celles du discours 
musical, avec les tentatives de E. Tarasti ou L. Tatit, reflètent clairement cette ouverture de la sémiotique sur le non-verbal. À cet égard, Greimas et son École reconduisent la tradition hjelmslevienne en reconnaissant la non-pertinence de la substance : la nature de la substance n'est pas définitoire pour la sémiotique :

La classification selon les canaux de transmission de signes (ou selon les ordres de sensation) repose sur la prise en considération de la substance de l'expression : or celle-ci n'est pas pertinente pour une définition de la sémiotique (qui est en premier lieu une forme) (Greimas et Courtés 1979 : 341).

À notre avis, s'il existe une continuité entre Hjelmslev et Greimas, c'est ici qu'il faut la chercher. Plus précisément, il faut voir que la glossématique de Hjelmslev se voulait, non pas une sémiotique générale, mais bien une sémiotique universelle. L'opposition " général » vs « universel » est un tournant décisif chez le sémioticien danois. C'est ce qui permet de distinguer le projet glossématique de sa préhistoire que l'on retrouve dans les Principes de grammaire générale ou dans La catégorie des cas. Car si Hjelmslev a commencé par viser le " général », c'est qu'il avait conscience d'appartenir à une tradition, comme celle de son compatriote R. Rask, ou celle de Grimm et Saussure. Le mot "général» revenait souvent dans le discours d'un grand nombre de linguistes de cette époque : en témoignent, par exemple, Linguistique historique et linguistique générale de A. Meillet, Cours de Linguistique générale de $\mathrm{F}$. de Saussure, Linguistique générale et linguistique française de $\mathrm{Ch}$. Bally. Beaucoup de passages des textes de Hjelmslev permettent de trancher cette question du "général» et de l'«universel». D'un texte de 1939, nous proposons le passage suivant :

Une grammaire générale ne se fait pas en alléguant quelque principe extralinguistique, qu'il soit d'ordre logique, psychologique, biologique, sociologique, ou qu'il soit emprunté à telle théorie philosophique ou à telle autre. Une grammaire générale ne se fait pas non plus par les généralisations prématurées de la squinting grammar, et la grammaire générale ne se confond pas avec la grammaire grécolatine, ni avec celle de l'indo-européen primitif. La grammaire générale ne s'acquittera de sa tâche qu'à condition de devenir grammaire comparative. C'est la comparaison des langues qui permet d'établir la grammaire du langage (Hjelmslev $1939: 140)$.

On est ici à l'opposé absolu de la théorie glossématique que Hjelmslev produira quelques années plus tard ${ }^{10}$, en quittant le général et l'induction pour l'universel et la déduction. Ici donc seule la méthode comparatiste est jugée pertinente pour établir une grammaire générale, pour saisir la réalisation d'un fait réalisable derrière le réalisé. L'exigence à laquelle est soumise la grammaire générale consiste à s'interroger sur les faits réalisables et surtout sur les conditions intrinsèques de leur réalisation. Ces conditions permettent de considérer l'objet de l'analyse sans aucune référence à la réalité mondaine, sans aucune référence extrinsèque, seule la langue fait référence, comme le dit clairement le principe d' « immanence» :

La grammaire générale ne se confond pas avec la grammaire universelle: la grammaire générale est faite par la reconnaissance des faits réalisables et des conditions immanentes de leur réalisation (ibid. ; nous soulignons).

21 Et plutôt que de rester à l'intérieur du signe, comme on l'a vu ci-dessus, Hjelmslev propose d'aller vers le texte avec une méthode déductive - dans l'acception particulière que ce terme a chez lui ${ }^{11}$-. Ainsi pour l'objet de la glossématique, il propose ceci :

Nous exigeons par exemple de la théorie du langage qu'elle permette de décrire non-contradictoirement et exhaustivement non seulement tel texte français donné, mais aussi tous les textes français existants, et non seulement ceux-ci, mais encore 
tous les textes français possibles et concevables - même ceux de demain, même ceux qui appartiennent à un avenir non déterminé - aussi longtemps qu'ils seront de même nature que les textes considérés jusqu'ici (1943 : 32). les mêmes chemins, mais qu'elles ne se dirigent pas vers le même terme, car leur objet de connaissance est différent. D'où la conclusion suivante: les réflexions du Hjelmslev linguiste, n'ont pas beaucoup impressionné Greimas; c'est plutôt le Hjelmslev épistémologue qui, semble-t-il, l'a fasciné, ainsi que le laisse voir la question des sémiotiques spécifiques.

\section{Les sémiotiques d'après Hjelmslev et Greimas}

Hjelmslev, dont l'œuvre est un maillon indispensable pour comprendre l'évolution de la sémiotique moderne, avait précisément pour dessein de formuler une sémiotique 
universelle qu'il a baptisée glossématique. Cependant son projet ne s'arrête pas là, on y trouve aussi une place considérable pour ce qu'on peut appeler des "sémiotiques spécifiques ", c'est-à-dire des sémiotiques qui se définissent par la singularité de leur champ de validité.

Ces sémiotiques spécifiques n'ont néanmoins bénéficié que d'un chapitre de douze pages dans les Prolégomènes ${ }^{14}$, alors que l'intérêt porté à ces sémiotiques par des chercheurs aussi divers que Barthes, Greimas, Metz et Kerbrat-Orecchioni ${ }^{15}$, pour ne citer que ceux$\mathrm{ci}$, est redevable en grande partie à ces quelques pages.

Sauf erreur de notre part, le nombre de types différents de ces sémiotiques spécifiques se limite à dix. Pour la commodité du lecteur, citons à nouveau leurs définitions. Avant de procéder à cette présentation, on doit souligner que ces dix sémiotiques spécifiques ont été distinguées les unes des autres sur une nouvelle base, à savoir l'opposition " sémiotique scientifique » vs « sémiotique non scientifique » :

1. Une métasémiotique est une sémiotique scientifique dont un ou plusieurs plans sont une sémiotique.

2. Une sémiotique connotative est une sémiotique non scientifique dont l'un des deux plans, celui de l'expression, est une sémiotique.

3. Une sémiotique dénotative est une sémiotique dont aucun des deux plans n'est une sémiotique.

4. Une métasémiotique scientifique est une métasémiotique dont l'objet est une sémiotique scientifique.

5. Une sémiologie est une métasémiotique dont la sémiotique objet est une sémiotique non scientifique.

6. Une sémiologie interne est une sémiologie dont la sémiotique objet est une sémiotique dénotative.

7. Une sémiologie externe est une sémiologie dont la sémiotique objet est une sémiotique connotative.

8. Une métasémiologie est une métasémiotique scientifique dont les sémiotiques objets sont des sémiologies.

9. Une métasémiologie interne est une métasémiotique dont les sémiotiques objets sont des sémiologies internes.

10. Une métasémiologie externe est une métasémiologie dont les sémiotiques objets sont des sémiologies internes.

30 Devant cette panoplie de sémiotiques, le lecteur aperçoit sans aucun doute l'importance du rôle accordé au mot "scientifique ", terme dangereux et lourd de connotations diverses. Qu'est ce que donc «scientifique " peut bien vouloir dire dans ce contexte? Essayons de le comprendre en suivant pas à pas le texte de Hjelmslev.

31 Selon les Prolégomènes, il s'agit d'un concept auquel incombe la fonction de distinguer deux types de sémiotiques. Sa définition est donc tributaire de la notion d'opération, concept clé dans la terminologie de la glossématique. Prenons pour exemple la métasémiotique que Hjelmslev qualifie de scientifique : c'est une opération, alors que la sémiotique connotative n'en est pas une. Mais qu'est ce qu'une opération? Hjelmslev la définit comme une « description en accord avec le principe d'empirisme » $(1943: 50)$.

Cette définition manifeste l'un des traits essentiels du style des Prolégomènes, où rien n'est démontré, rien n'est défini dans le sens traditionnel du terme. L'axiomatique glossématique n'est définie que relationnellement; il y a une détermination entre les 
définitions, celles qui précèdent présupposant celles qui suivent et inversement. Tout cela ressemble à une pelote telle que si l'on tire un fil, tout le reste suit. Et puisque les choses fonctionnent de la sorte, poursuivons avec la notion d'empirisme :

La description devra être exempte de contradiction, exhaustive et aussi simple que possible (Hjelmslev 1985 : 88).

À partir de là, on peut inférer que les sémiotiques scientifiques sont régies par le principe d'empirisme, tandis que les sémiotiques non scientifiques ne le sont pas.

De ce fait, on voit que la scientificité est tributaire de l'opération, et que l'opération est une propriété du principe d'empirisme. Ainsi les sémiotiques connotatives, comme sémiotiques non scientifiques, peuvent entraîner la contradiction, ne sont pas exhaustives et donnent des résultats qui enfreignent le principe de simplicité, tandis que la sémiotique dénotative est par principe non contradictoire et tend vers l'exhaustivité et la simplicité.

De ce point de vue, le concept d'opération est conforme à l'adage hjelmslevien suivant lequel tous les concepts sont définis comme étant des concepts souples et relatifs ${ }^{16}$.

Tout se résume donc en ceci : comme les principes d' "analyse », de "grandeur », de « signe », de «signification » et d'« empirisme » sont relatifs, le concept d'opération aussi ne peut être que simple et relatif :

De telles définitions font de l'opération et de la procédure des concepts souples

(1943: 50).

37 Cela signifie que ce qui sépare les sémiotiques scientifiques et les sémiotiques non scientifiques est poreux ; dès lors, il n'y a pas de division absolue entre ces deux sémiotiques. À bien les examiner, cependant, les sémiotiques scientifiques et les sémiotiques non scientifiques vont en sens inverse les unes des autres. Pour saisir ce qui les distingue, glossématiquement, nous choisirons, faute de pouvoir embrasser la totalité des sémiotiques, deux sémiotiques distinctes se rapportant chacune à une classe précise de sémiotiques. Parmi les sémiotiques scientifiques, on interrogera la sémiotique dénotative, et parmi les sémiotiques non scientifiques, la sémiotique connotative.

À partir de ces deux types de sémiotiques, nous pouvons voir que lorsque Hjelmslev parle de sémiotique non scientifique, cela ne veut pas dire, comme semblent le comprendre Greimas et Courtés, qu'elle est située "en dehors du champ de la scientificité ", mais tout simplement qu'elle n'est pas une opération, et par conséquent, pas une théorie. Voilà le critère qui nous semble décisif pour distinguer ces deux sémiotiques. En d'autres termes, si ces deux sémiotiques sont opposées selon le critère de scientificité, il faut préciser que celui-ci est lui-même dépendant d'un autre principe, le principe d'empirisme - critère indispensable pour parler du concept de théorie. En effet, qu'est-ce qu'une théorie, demande Hjelmslev, sinon une voie qui nous conduit «à des résultats conformes aux faits d'expérience» (1943: 50)?

Revenons aux deux exemples choisis ci-dessus, sémiotique dénotative vs sémiotique connotative, pour voir les choses sous un angle un peu différent. Pour la sémiotique connotative, il s'agit d'une sémiotique qui fonde en constante ce qui est susceptible de variation dans la sémiotique dénotative. C'est donc la variabilité du plan d'expression de la sémiotique dénotative qui rend possible l'apparition d'une sémiotique connotative. C'est à partir de là qu'il devient aisé de suivre la pensée du maître danois.

Premièrement, on peut constater que la sémiotique dénotative est définie comme théorie, c'est-à-dire comme sémiotique exempte de contradiction, exhaustive et simple, tandis 
que la sémiotique connotative est vue comme hiérarchie «dont chacune des composantes admet une analyse ultérieure en classe définie par relation mutuelle, de telle sorte que chacune de ces classes admette une analyse en dérivés définis par relation mutuelle » (Hjelmslev 1985 : 96).

41 Almeida voit ce problème des sémiotiques scientifiques et des sémiotiques non scientifiques autrement et permet, à notre avis, de comprendre clairement cette opposition. Il propose d'appréhender les sémiotiques scientifiques comme des sémiotiques décrivantes, et les sémiotiques non scientifiques comme décrites:

Tout se résume donc en ceci : une sémiotique non scientifique est une hiérarchie décrite, et une sémiotique scientifique est la même hiérarchie mais prise en tant que décrivante (1998:3).

Un doute s'élève. La sémiotique dénotative est une sémiotique dont aucun des deux plans n'est une sémiotique, c'est pourquoi elle est décrivante, tandis que la plan d'expression de la sémiotique connotative est une sémiotique, et c'est pourquoi elle est vue comme décrite, c'est-à-dire comme hiérarchie, à l'encontre de la décrivante qui est une opération, et par voie de conséquence, une théorie scientifique. Or cela ne veut pas dire que la sémiotique connotative est dépourvue de scientificité, mais simplement qu'elle n'est pas une théorie parce qu'elle dépend d'un principe souple et relatif, le principe d'opération.

Le seuil qui sépare, donc, les sémiotiques scientifiques des sémiotiques non scientifiques est fragile, puisqu'il dépend d'un moule souple et maniable selon les procédures d'analyse.

En gardant en mémoire la typologie hjelmslevienne, Greimas propose de diviser les sémiotiques en trois niveaux, sans pour autant faire intervenir le critère de scientificité, écarté au profit du nombre des plans dont une sémiotique est constituée : sémiotique monoplane, sémiotique biplane, sémiotique pluriplane ${ }^{17}$.

Les premières, c'est-à-dire les monoplanes, sont les systèmes de symboles dont les deux plans du langage sont liés par une relation de conformité. C'est le cas de l'algèbre et des jeux. À la différence des autres sémiotiques, les biplanes, ou sémiotiques proprement dites pour Hjelmslev, sont celles qui comportent deux plans. Quant aux sémiotiques pluriplanes, Greimas entend les sémiotiques biplanes dont au moins un des deux plans est une sémiotique. Les sémiotiques connotatives et les métasémiotiques font partie de cette troisième catégorie.

Ensuite Greimas abandonne cette tripartition en proposant de distinguer les sémiotiques linguistiques des sémiotiques non linguistiques.

Cette dernière opposition le conduit à reconnaître que le phénomène de la signification « peut se cacher sous toutes les apparences sensibles, elle est derrière les sons, mais aussi derrière les images, les odeurs et la saveurs, sans pour autant être dans les sons ou dans les images» (Greimas 1971: 49), d'où l'indifférence de la forme sémiotique à la nature de la substance qui la manifeste.

Ceci posé, l'on peut tout de même prévoir des cas où il y ait hétérogénéité des sémiotiques: il sera alors possible d'examiner des sémiotiques élaborant leur plan de l'expression à partir d'éléments relevant de sémiotiques diverses. C'est ce que Greimas appelle sémiotiques syncrétiques. Qu'il suffise d'évoquer, par exemple, tout le travail réalisé par la sémiotique du cinéma, la proxémique et la sémiotique visuelle où se manipulent des éléments provenant de plusieurs sémiotiques différentes. 
, il convient d'ajouter que ces sémiotiques spécifiques constituent un champ de recherches qui procèdent souvent par emprunts conceptuels. S'appropriant telle ou telle notion, qui a pris naissance dans la sémiotique universelle, les sémiotiques spécifiques la modèlent et la redéfinissent conformément à leurs principes de pertinence. Ce fut le cas de la sémiotique visuelle, ce fut aussi le cas de la sémiotique musicale ou architecturale ; ou celui encore de la sémiotique des passions, du goût et des odeurs. C'est enfin le cas aujourd'hui pour la sémiotique cognitive qui bat son plein avec P. Ouellet et P. A. Brandt. Des propos de Greimas et Courtés, pleins d'optimisme et d'ambition, confirment ce point de vue :

Toutes ces distinctions et réorganisations, mêmes si elles introduisent parfois quelques confusions dans le champ sémiotique, sont à considérer comme un signe de santé et de vitalité d'une sémiotique qui se veut un projet de recherche en train de se faire (1979: 344$)$.

À partir de ce fonds épistémologique commun constitué par des principes comme celui de l'universalisme ou celui des sémiotiques spécifiques, il est clair que chaque sémioticien développe sa propre problématique. Greimas est donc loin d'être le continuateur de Hjelmslev. Les deux sémiotiques sont différentes. On comprend dès lors que ni Hjelmslev ni Greimas ne visent à proprement parler à rendre compte du texte, car leurs théories sont dépourvues de toute textualité ${ }^{18}$. Si le premier cherchait le système derrière le texte, le second voulait accéder aux structures logico-grammaticales sous-jacentes au niveau discursif manifeste. Cette divergence reconnue, même par Greimas, se manifeste essentiellement aujourd'hui par les ouvertures de la sémiotique sur d'autres horizons. Si, durant toute l'ère structuraliste, la sémiotique de Greimas a abordé le texte d'un point de vue discontinu (à travers l'heuristique du parcours génératif, avec ses niveaux et ses composantes), en revanche, actuellement, c'est le continu qui apparaît sur le devant de la scène, renvoyant au passé l'immense puzzle théorique construit sur plus de vingt-cinq ans.

51 Aujourd'hui plus que jamais, la sémiotique greimassienne cherche ses marques. Certains parlent d'une nouvelle sémiotique, du maitre dépassé ou trahi : tout cela montre que la sémiotique existe ${ }^{19}$. Les séminaires, les congrès et les publications en témoignent clairement. Car si le continu a permis à la sémiotique d'éviter les ressassements et l'immobilisme en remettant en question l'autonomie du langage, il a conduit les recherches vers l'«allonomie», pour reprendre une expression de Varela, vers la perception, l'intentionnalité et le corps, bref vers la cognition. Or, comme l'a noté Rastier, la cognition « reste un objet dont aucune science n'a encore su déposséder la philosophie » (1991: 20), et comme aucune science parmi les recherches cognitives ne prend la cognition comme objet spécifique, pourquoi donc la sémiotique resterait-elle à l'écart de ce programme interdisciplinaire ${ }^{20}$ ? Mais ce n'est pas parce que la sémiotique peut être mise au nombre des recherches cognitives, avec les travaux éminents de Petitot, Brandt et Ouellet, qui donnent plus de dynamique - c'est le mot en vogue ces derniers temps - à l'heuristique sémiotique, qu'on doit faire tabula rasa de l'immense puzzle de la sémiotique du discontinu. Car si maintenant «ça commence à bouger», il nous faudra préciser à quelles conditions la sémiotique peut participer à ce débat sur la cognition. Nous resterons sur cette interrogation. 


\section{BIBLIOGRAPHIE}

Ablali D. [à paraître] : « Hjelmslev, Greimas, Rastier : une continuité impossible autour de la notion de texte » in Actes du colloque Sémio 2001. Des théories aux problématiques, Limoges, Pulim. Almeida I. 1998 : « Le style épistémologique de Hjelmslev » in http: //www.msh-paris.fr/texto/. Auroux S. 1988 : « La notion de linguistique générale » in Histoire, épistémologie, langage, 10-II : 37-56.

Arrivé M. 1981 : « La glossématique » in R. Posner \& J.N. Green (dir.), Trends in Romance Linguistics and Philology, tome 2, La Haye, Mouton.

- 1982 : « Hjelmslev lecteur de Martinet lecteur de Hjelmslev » in Linx,6 : 77-93 ; réédité in Hjelmslev $1985:$ 195-207.

-2000 : «Saussure, Barthes, Greimas » in Modèles linguistiques, 41 : 19-37.

— \& Ablali D. 2001 : « Hjelmslev et Martinet : correspondance, traduction, problèmes théoriques » in La linguistique, 37-1.

Badir S. 1998 : «La notion de texte chez Hjelmslev » in http: //www.msh-paris.fr/texto/.

-2000 : Hjelmslev, Paris, Belles lettres.

Barthes R. 1964 : « Éléments de sémiologie » in Communication, 4.

Bouquet S. 1997 : Introduction à la lecture de Saussure, Paris, Payot.

- [à paraître] : « De l'hexagramme cognitiviste à une sémiotique de l'interprétation », in Actes du colloque Sémiotique des cultures et sciences cognitives, dirigé par S. Bouquet et Fr. Rastier.

Brandt P. A. 2000 : « De la linguistique structurale à la linguistique cognitive. Avec Hjelmslev », in http : //www.hum.au.dk/semiotics/docs, 12 p.

Fischer-Jorgensen E. 1997 : « Hjelmslev et le cercle linguistique de Copenhague », in A. Zinna (éd.), Hjelmslev aujourd'hui, Turnhout, Brépols, 25-36.

Fontanille J. 1998 : Sémiotique du discours, Limoges, PULIM.

- 2001 : Les modèles sémiotiques, document de travail, Séminaire Intersémiotique 2000-2001.

Greimas A. J. 1966 : Sémantique structurale, Paris, PUF.

- 1970 : Du Sens, Paris, Seuil.

- 1974 : «Entretien » in Le Monde (6 juin).

- 1983 : Du Sens II, Paris, Seuil.

- 1986 : « Discussion » avec A. Zinna, in Louis Hjelmslev, linguistica e Semiotica structurale, Versus, $43: 45-57$.

- 1987 : «A.J. Greimas mis à la question » in M. Arrivé \& J-Cl. Coquet (éds), Sémiotique en jeu, Paris, Amsterdam, Hadès-Benjamins, 301-330.

- 1987 : De L’imperfection, Périgueux, Fanlac.

— \& Courtés J. 1979 : Sémiotique. Dictionnaire Raisonné de la Théorie du Langage, Paris, Hachette. 
— \& Ricoeur P. 1989 : « Débat » in A. Hénault, Le Pouvoir comme passion, Paris, P.U.F., 195-216.

— \& Fontanille J. 1991 : Sémiotique des passions, Paris, Seuil.

Hjelmslev L. 1929 : Principes de grammaire générale, Copenhague, Bianco Lunos Bogtrykkeri.

- 1939 : « La structure morphologique » in Essais linguistiques, Paris, Minuit, p. 122-147.

- 1943 : Prolégomènes à une théorie du langage, Paris, Minuit, 1968.

- 1971 : Essais linguistiques, Paris, Minuit.

- 1985 : Nouveaux essais, Paris, P.U.F.

Martinet A. 1946 : « Au sujet des fondements de la théorie linguistique de Louis Hjelmslev » in BSL , 124-125/1-2:19-42.

Normand Cl. 1993 : « Les Principes de grammaire générale : genèse d'un modèle abstrait » in Hjelmslev et la sémiotique contemporaine. Travaux du cercle linguistique de Copenhague, 65-77.

Rasmussen M. 1993 : « La situation de l'observation en linguistique. Une comparaison entre Louis Hjelmslev et Niels Bohr » in Hjelmslev et la sémiotique contemporaine, Travaux du cercle linguistique de Copenhague, 112-130.

Rastier Fr. 1985 : «L'œuvre de Hjelmslev aujourd'hui » in: Louis Hjelsmelv, Linguistica, semiotica, epistemologia. Pratogora, 7-8 : 109-126.

- 1987 : Sens et textualité, Paris, Hachette.

- 1989 : «De l'universalisme en linguistique » in Linguistique et sémiotique : actualité de Viggo

Brøndal. Travaux du cercle linguistique de Copenhague, 95-106.

- 1991 : Sémantique et recherches cognitives, Paris, PUF.

- 1997 : « Les fondations de la sémiotique et le problème du texte. Questions sur les Prolégomènes » in Zinna A. (éd.), Hjelmslev aujourd'hui, Turnhout, Brépols, 141-161.

Saussure F. de 1916 : Cours de linguistique générale, Paris, Payot, 1991.

- 1968-1974 : Cours de linguistique générale, Wiesbaden, Otto Harrassowitz, édition critique par R.

Engler, 2 tomes.

Varela Fr. J. 1989 : Autonomie et connaissance. Essais sur le vivant, Paris, Seuil.

Zilberberg Cl. 1987 : Raison et poétique du sens, Paris, P.U.F.

Zilberberg Cl. 1997 : « Une continuité incertaine : Saussure, Hjelmslev, Greimas » in Zinna A. (éd.), Hjelmslev aujourd'hui, Turnhout, Brépols, 165-192.

\section{NOTES}

1. La dimension linguistique de la sémiotique de Greimas est matière à réflexion. S'agit-il vraiment d'une sémiotique linguistique? P. A. Brandt en dit ceci : « Ni la discursivité, ni la narrativité, ni la catégorie relationnelle ne semblent dérivables de ce que l'on peut savoir sur la phrase ou sur les morphologies grammaticales, trop près des mots pour instruire la connaissance du contenu discursif. La linguistique demeure une référence méta-théorique, presque nostalgique » $(2000: 3)$.

2. Semiotics n'est un pluriel que d'un point de vue morphologique; sémantiquement et syntaxiquement il fonctionne comme non comptable. En français, il faudrait traduire par « de la sémiotique », « dans le domaine sémiotique ». 
3. Il faut dire que le développement de la sémiotique française d'obédience greimassienne, doit beaucoup, après la mort de Greimas, à J. Fontanille. La création du séminaire « inter-sémiotique » au CNRS appelant à la collaboration interdisciplinaire et interthéorique témoigne clairement de ses efforts en vue d'effacer le discrédit attaché à cette sémiotique, considérée comme trop « fermée », « dogmatique » et " chapellisée ».

4. Un autre sémioticien comme $\mathrm{Cl}$. Zilberberg en dit ceci : "Comment saluer la rigueur de Hjelmslev et ne pas s'étonner, s'inquiéter, du même coup, du peu d'audience, ou de l'audience insuffisante de son œuvre. C'est moins Hjelmslev qui est ici accusé que la légèreté ordinaire des commentateurs » (Zilberberg $1988: 4$ ).

5. Ce centenaire a été par contre célébré en Italie, plus précisément à l'université de Padoue, sous la direction de R. Galassi au mois d'octobre 1999.

6. Après bien sûr le long compte-rendu des Prolégomènes fait en 1946 par Martinet dans le Bulletin de la Société Linguistique. Pour plus d'information sur ce sujet, cf. Arrivé \& Ablali 2001.

7. «Une continuité incertaine : Saussure, Hjelmslev, Greimas », cf. Zilberberg 1997.

8. Cf. Greimas \& Courtés $1979: 103$.

9. Sur cette question de "général ", on peut se référer à Auroux 1988, à Normand 1993, et à propos de cette notion chez Saussure, on trouvera une étude détaillée dans Bouquet 1997 : 173-178; 239-248, de même que l'on peut lire avec intérêt l'article de Rastier (1989), sur l'universalisme.

10. À cet égard, il ne serait peut-être pas inutile de rappeler que de la parution des Prolégomènes, l'ouvrage le plus lu de Hjelmslev, il n'a jamais été question dans les correspondances de celui-ci, contrairement à l'ouvrage projeté dès les premiers jours de 1936 par Hjelmslev et Uldall, et qui ne verra le jour que sous la signature de Uldall, Outline of Glossematics, avec une préface de Hjelmslev.

11. Pour un supplément d'information, cf. Badir 1998.

12. Elle en dit ceci : «Hjelmslev ne s'imposait pas aux autres. Mais quand Diderchsen, s'est proposé d'appliquer la glossématique à la description de la langue danoise, il a salué ce projet avec joie et gratitude. Seulement il ne considérait pas comme sa tâche de donner des leçons de répétition sur sa théorie. Il avait mis sa théorie à la disposition des linguistes, et c'est à eux de l'appliquer » (1997: 32).

13. À cet égard, on peut consulter Badir 1998, Rastier 1997 ou encore Ablali 2001.

14. Il s'agit du chapitre vingt-deux des Prolégomènes, intitulé «Langages de connotation et métalangages ".

15. À cet égard, nous ne pouvons que renvoyer chaudement le lecteur au livre de S. Badir, Hjelmslev, qui aborde, bien armé, cette question de la réception de la notion de « connotation », et son emploi en France dans un sens différent que celui que lui donne Hjelmslev.

16. Voici quelques exemples :

Nous avons défini l'analyse de telle sorte que rien n'y indique si elle est simple ou continue. [...] Le concept d'analyse est souple (Hjelmslev 1943 : 49).

Toute grandeur, et par conséquent tout signe, est définie de façon relative et non absolue (id. : 67). Dans tous les cas, on comprend la signification comme une notion relative (ibid.; nous soulignons).

17. Pour un supplément d'information, cf. l'entrée « sémiotique » dans Greimas \& Courtés 1979: 342.

18. Le terme de textualité est pris ici dans le sens que lui donne Rastier, et qui consiste « en un ensemble de propriétés de cohésion et de cohérence qui rendent un texte irréductible à une suite d'énoncés » $(1987: 281)$.

19. Quoique Fontanille pense que le statut de la sémiotique n'est pas en vogue, aujourd'hui, dans les sciences humaines : "Il semblerait qu'une "pause théorique » (comme on dit une "pause 
descriptive » en narratologie) soit aujourd'hui nécessaire. En effet, d'un côté, la théorie, ses modèles, et ses exigences, n'est pas en vogue dans les sciences humaines d'aujourd'hui... » (2001). 20. Tout le propos du colloque "Sémiotique des cultures et sciences cognitives", organisé par S. Bouquet et Fr. Rastier, et dont les actes ne tarderont pas à paraître, a été précisément cette question, à laquelle une réponse apparaît, validée par les psychologues (Bruner), ethnologues (Geertz), éthologues (Cyrulnik), etc. qui ont participé à ce colloque: la sémiotique peut, précisément, être considérée et définie comme une autre forme d'interdisciplinarité que le courant cognitiviste. Ce point de vue est synthétisé dans l'article de S.Bouquet, «De l'hexagramme cognitiviste à une sémiotique de l'interprétation", placé pour cette raison en introduction de ses actes.

\section{RÉSUMÉS}

Nous proposons d'étudier dans cet article la coexistence au sein de la sémiotique européenne de deux paradigmes, attachés respectivement aux noms de Hjelmslev et de Greimas. Plus précisément, nous cherchons à savoir ce qu'il en est de la reconduction du projet glossématique de Hjelmslev dans la sémiotique de Greimas. Nous essayerons de montrer que ce sentiment de parenté n'est pas pour autant sans fondement: entre Hjelmslev et Greimas, il y a bien une continuité. Seulement, il ne faut pas la chercher directement dans la reconduction par Greimas du projet linguistique de la glossématique, mais bien dans la réflexion épistémologique de Hjelmslev, lorsque celui-ci conçoit la sémiotique comme une sémiotique universelle avec des sémiotiques spécifiques.

The aim of this paper is to study, In European semiotics, two paradigms generally related and respectively known as Hjelmslev's and Greimas'. More precisely we shall try to make clear that Hjelmslev's glossematic project is to be recognized in some degree in Greimas'semiotics.This relationship however does not appear in a renewal by Greimas of the glossematic project ; the real continuity is to be found in Greimas' interest for Hjelmslev's epistemological thinking, particularly when the latter conceived semiotics as combining universal character and specific fields.

\section{AUTEUR}

\section{DRISS ABLALI}

UMR : Modyco, 2329 CNRS/Paris, GRHEL, Université de Paris-X-Nanterre, driss.ablali@uparis10.fr 